\title{
Deposition of Indium Nanoparticles on Powdered Material by Pulse Arc Plasma to Synthesize Catalysts for Friedel-Crafts Alkylation*
}

\author{
Satoru Yoshimura ${ }^{\dagger}$ \\ Center for Atomic and Molecular Technologies, Graduate School of Engineering, \\ Osaka University, 2-1 Yamadaoka, Suita, Osaka 565-0871, Japan \\ Yoshihiro Nishimoto \\ Department of Applied Chemistry, Graduate School of Engineering, Osaka University and \\ Frontier Research Base for Young Researchers, Graduate School of Engineering, \\ Osaka University, 2-1 Yamadaoka, Suita, Osaka 565-0871, Japan \\ Satoshi Sugimoto \\ Center for Atomic and Molecular Technologies, Graduate School of Engineering, \\ Osaka University, 2-1 Yamadaoka, Suita, Osaka 565-0871, Japan \\ Masato Kiuchi \\ Center for Atomic and Molecular Technologies, Graduate School of Engineering, \\ Osaka University, 2-1 Yamadaoka, Suita, Osaka 565-0871, Japan and \\ National Institute of Advanced Industrial Science and Technology (AIST), \\ 1-8-31 Midorigaoka, Ikeda, Osaka 563-8577, Japan. \\ Makoto Yasuda \\ Department of Applied Chemistry, Graduate School of Engineering, \\ Osaka University, 2-1 Yamadaoka, Suita, Osaka 565-0871, Japan \\ (Received 14 December 2017; Accepted 6 March 2018; Published 26 April 2018)
}

\begin{abstract}
Indium nanoparticles were deposited on zeolite powders using a pulse arc plasma system. The resulting materials were capable of catalyzing an organic chemical reaction, namely the Friedel-Crafts alkylation between benzhydryl chloride and anisole. Varying the electrical capacitance in the plasma system $(360 \mu \mathrm{F}$ or $1080 \mu \mathrm{F})$ resulted in some changes in the indium concentration on the sample surface and in the size of the indium nanoparticles, but the catalytic ability of the material was largely unaffected. [DOI: 10.1380/ejssnt.2018.105]
\end{abstract}

Keywords: Catalysis; Indium oxide; Powders; Plasma processing

\section{INTRODUCTION}

Indium has attracted much attention with regard to its catalytic applications [1-3]. As an example, indium oxide powders have been reported to be capable of catalyzing various chemical reactions [4-6]. However, commercially available indium oxide powders are insoluble in organic solvents, which limits their catalytic activity because of a lack of active surface area. Indium oxide nanoparticles deposited on powdered materials should provide large surface areas and therefore numerous active sites, both of which would be expected to enhance catalysis.

Previously, we successfully synthesized a catalyst by exposing silicon dioxide films to an indium ion beam, using a low energy ion beam system [7-9]. Subsequently, we attempted to implant indium atoms in powdered materials using the same ion beam system, using pellets produced by compression molding. In this system, the pellet was installed in a sample holder and then exposed to an indium ion beam. However, the indium ions were simply deposited on the surface of the pellet to form a film of metallic indium. This failure to insert indium homoge-

\footnotetext{
* This paper was presented at the 8th International Symposium on Surface Science, Tsukuba International Congress Center, Tsukuba, Japan, October 22-26, 2017.

† Corresponding author: yosimura@ppl.eng.osaka-u.ac.jp
}

neously throughout the entire pellet resulted in a lack of catalytic activity [10].

Agawa et al. [11] reported that carbon powder exposed to platinum nanoparticles generated using a pulse arc plasma system [12] exhibits catalytic activity. In this prior work, the carbon powder was agitated with scrapers during the irradiation, allowing the uniform insertion of the platinum nanoparticles. A pulse arc plasma system can also be used to generate small, uniformly dispersed particles of indium for deposition on a powder surface, so we attempted to employ this process to synthesize an indium-based catalyst. We selected a zeolite powder as the target and demonstrated that an indium-doped zeolite will catalyze the Friedel-Crafts alkylation reaction between benzhydryl chloride and anisole [13, 14]. In our prior work, specimens exposed to indium nanoparticles were removed from the vacuum chamber of the pulse arc plasma system under ambient air, and this process could have oxidized the indium particles on the zeolite [15]. The resulting indium oxide particles may therefore have been responsible for catalyzing the Friedel-Crafts alkylation. In fact, we also demonstrated that a commercially available indium oxide powder was also capable of catalyzing the same reaction [15].

Following on from the above work, the present study examined whether or not the properties of indium-doped zeolites are affected by the electrical capacitance of the capacitor used in the pulse arc plasma system. 


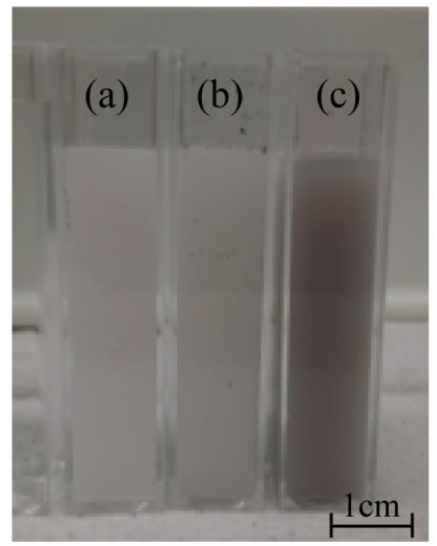

FIG. 1. Photographic images of (a) the pristine zeolite, and zeolite samples exposed to indium nanoparticles using (b) $360 \mu \mathrm{F}$ and (c) $1080 \mu \mathrm{F}$ capacitors. All samples were suspended in distilled water.

\section{EXPERIMENTAL PROCEDURES}

Indium irradiation experiments were carried out using a pulse arc plasma source (ADVANCE RIKO, Japan, model: APD-P). A schematic diagram of this device has been published in a previous report [16]. The vacuum chamber in the plasma system was evacuated with a turbomolecular pump and the pressure in the chamber during irradiation was $1 \times 10^{-3} \mathrm{~Pa}$. The arc plasma source consisted of a cylindrical anode and a cathode rod situated coaxially to one another. The cathode, a cylindrical insulator, and the trigger electrode were assembled in close proximity to one another in a concentric circular arrangement. Upon applying $70 \mathrm{~V}$ between the cathode and anode, a high voltage of $+3.4 \mathrm{kV}$ was sent to the trigger electrode via the insulator between the trigger electrode and cathode over a span of several microseconds. Electrons subsequently flowed over the insulator surface from the base of the cathode to the trigger electrode and the pulse arc discharge between the anode and cathode was sustained by the electric power stored in a capacitor, the capacitance of which could be varied. The capacitor was charged and discharged repeatedly, during which the cathode material was evaporated. As a result, nanoparticles of the cathode material were intermittently formed and imparted to a substrate placed just below the source. The nanoparticle injection energy was estimated to be approximately $35 \mathrm{eV}$ [17]. The dose of nanoparticles sent to the substrate could be controlled by varying the number of discharge repetitions. It has been demonstrated empirically that the size of these metallic nanoparticles is affected by the electrical capacitance of the capacitor used in the arc plasma system. Specifically, the mean diameter of nanoparticles deposited on the substrate increases along with the capacitance [18].

This work employed a powdered zeolite, molecular sieves $5 \AA$ (MS5A, Sigma-Aldrich Japan), as the target for the indium irradiation experiments. The molecular formula of MS5A is $\mathrm{Ca} /{ }_{n} \mathrm{Na}_{12-2 n}\left[\left(\mathrm{AlO}_{2}\right)_{12}\left(\mathrm{SiO}_{2}\right)_{12}\right]$ and the average particle size of the material was less than $10 \mu \mathrm{m}$. During these trials, a cylindrical container with a diameter of $8 \mathrm{~cm}$ was positioned immediately below the plasma source and filled with a $12 \mathrm{~g}(20 \mathrm{~mL})$ portion of
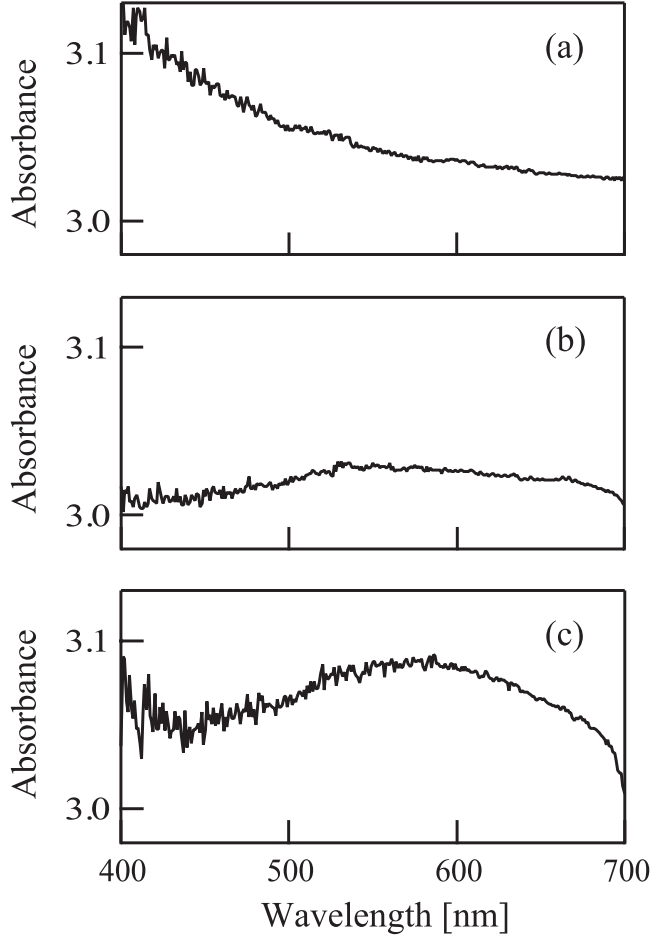

FIG. 2. Visible absorbance spectra of (a) the pristine zeolite, and zeolite samples exposed to indium nanoparticles using (b) $360 \mu \mathrm{F}$ and (c) $1080 \mu \mathrm{F}$ capacitors. All samples were suspended in distilled water.

the MS5A. Throughout the irradiation, two scrapers on the bottom of the container stirred the zeolite powder so that the entire mass was exposed to the nanoparticles in a uniform manner.

The cathode rod in the plasma source was made of metallic indium so as to obtain indium nanoparticles. Three samples were prepared for evaluations of surface properties and catalytic activities: (a) pristine zeolite, and zeolite exposed to indium nanoparticles using electrical capacitance values of (b) $360 \mu \mathrm{F}$ and (c) $1080 \mu \mathrm{F}$. Assuming that all the indium nanoparticles generated in the system were successfully deposited on the zeolite, the last two samples both contained $0.4 \mathrm{wt} \%$ indium.

The resulting samples were subsequently analyzed by visible light absorbance spectroscopy (SHIMADZU, Japan, model: UV-1800), X-ray diffraction (XRD, Rigaku, Japan, model: RINT2200), X-ray photoelectron spectroscopy (XPS, SHIMADZU, Japan, model: ESCA850), transmission electron microscopy (TEM, HITACHI, Japan, model: H8000) and scanning electron microscopy (SEM, HITACHI, Japan, model: S-4800). Finally, the catalytic abilities of the samples were assessed.

\section{EXPERIMENTAL RESULTS AND DISCUSSION}

Samples (a) to (c) were each separately placed in a test cell and suspended in pure water, as shown in Fig. 1(ac). The visible light absorbance of each suspension was determined using a spectrophotometer UV-1800 in transmission mode, with the results provided in Fig. 2(a-c). Sample (c) showed a slightly higher absorbance than sam- 


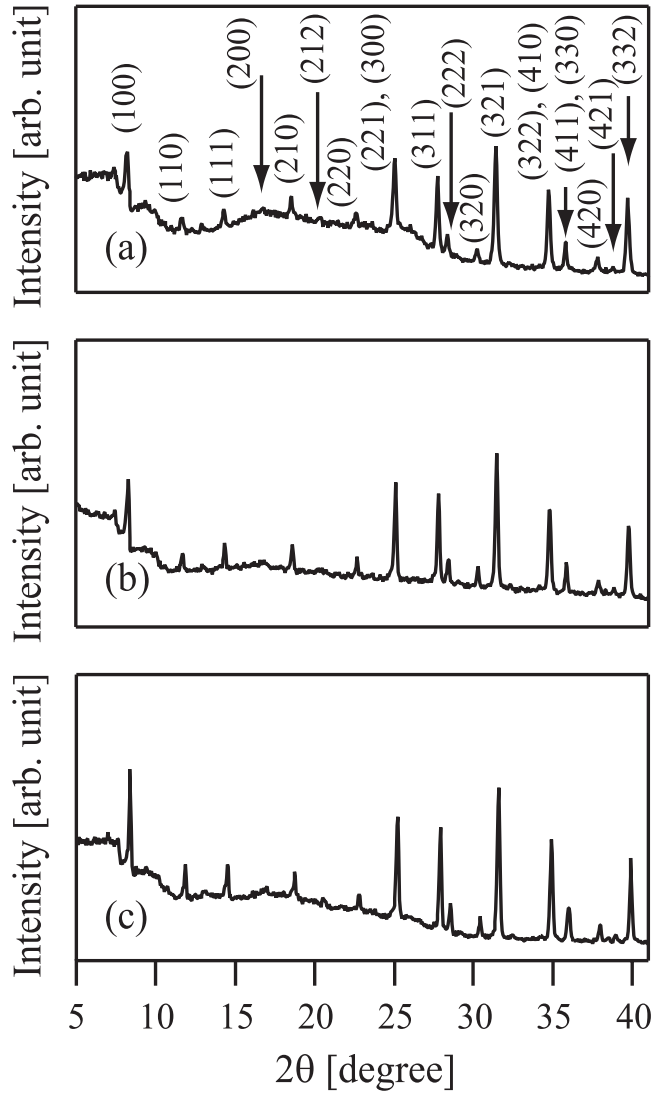

FIG. 3. X-ray diffraction patterns $(\theta-2 \theta$ method) obtained from (a) the pristine zeolite, and zeolite samples exposed to indium nanoparticles using (b) $360 \mu \mathrm{F}$ and (c) $1080 \mu \mathrm{F}$ capacitors. See Ref. [19] for the peak assignment in Fig. 3(a).

ple (a) and had a dark grey coloration [Fig. 1(c)] that was different from the white color of the untreated zeolite [Fig. 1(a)]. Figure 2(c) suggests the presence of an absorbance peak at approximately $600 \mathrm{~nm}$ but, because this peak is quite broad, it may result from diffuse reflectance that is largely independent of wavelength. Figure 1(b) shows that sample (b) remained white. This material also exhibited a slightly lower absorbance [Fig. 2(b)].

The indium-doped samples were analyzed by XRD using a diffractometer RINT2200 and the resulting patterns are presented in Fig. 3(b, c), along with the pattern obtained from a pristine zeolite sample in Fig. 3(a) for comparison purposes. The pattern in Fig. 3(a) is identical to a previously published pattern for this same material [19]. Peaks in the XRD pattern of the pristine zeolite sample have been already identified in the previous paper [19], assigned XRD peaks of which are shown in Fig. 3(a) for reference. All three patterns are similar, with no clear peaks corresponding to metallic indium or indium compounds in Fig. 3(b, c), possibly due to the wide dispersion of the nanoparticles.

The samples were subsequently analyzed by XPS. Each specimen was set in a sample holder and held in place with double-sided carbon tape, and the resulting spectra are shown in Fig. 4. These spectra exhibit peaks corresponding to the constituent atoms of MS5A (Ca, Na, $\mathrm{Si}$, and $\mathrm{Al}$ ) as indicated by the arrows in the figure, and the peak intensities of each of these elements are similar for each specimen. Note that the $\mathrm{C} 1 \mathrm{~s}$ peak in these

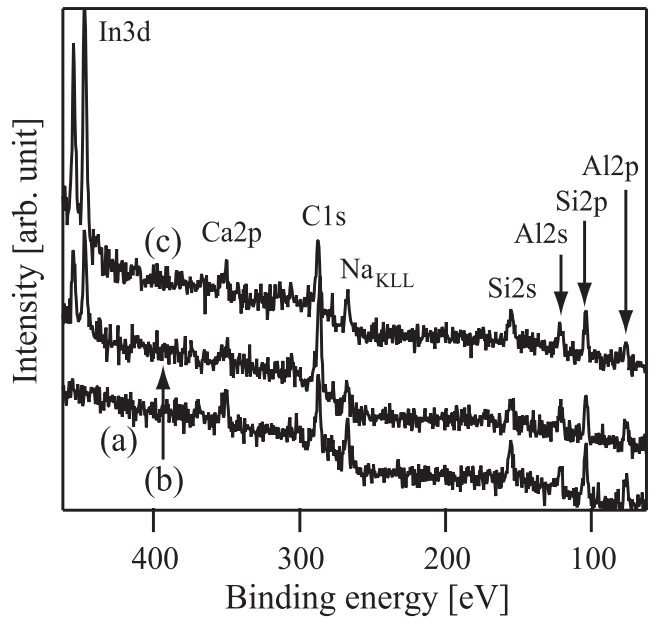

FIG. 4. X-ray photoelectron spectra acquired from (a) the pristine zeolite, and zeolite samples exposed to indium nanoparticles using (b) $360 \mu \mathrm{F}$ and (c) $1080 \mu \mathrm{F}$ capacitors.

spectra can be ascribed to the carbon tape. However, the spectra acquired from the indium-doped zeolites also contain two additional peaks corresponding to indium [In 3d; Fig. 4(b, c)]. Although no metallic indium or indium compound peaks were observed in the XRD patterns, the XPS data clearly show the presence of indium atoms on the sample surfaces. The In 3d peak intensity in Fig. 4(c) is also greater than that in Fig. 4(b). Although both specimens were injected with approximately the same mass of indium, the atomic concentrations of indium on the sample surfaces were determined to be $1.4 \%$ and $3 \%$ in samples (b) and (c), respectively, based on the XPS data. These results suggest that the retention of the nanoparticles on the zeolite surface is augmented as the electrical capacitance of the plasma system increases. The In $3 \mathrm{~d}$ peaks in Fig. 4(b, c) also demonstrate an energy shift from the metallic indium peak position, indicating the presence of $\mathrm{a}+3$ valence indium compound such as indium oxide.

The surface morphologies of the samples were assessed using TEM and a typical TEM image of an indium-doped zeolite [sample (b)] is shown in Fig. 5. Unfortunately, the sample was too thick to allow precise examination by TEM and it is difficult to identify indium nanoparticles on the surface. In addition, we found that the sample morphology was modified by the TEM electron beam at

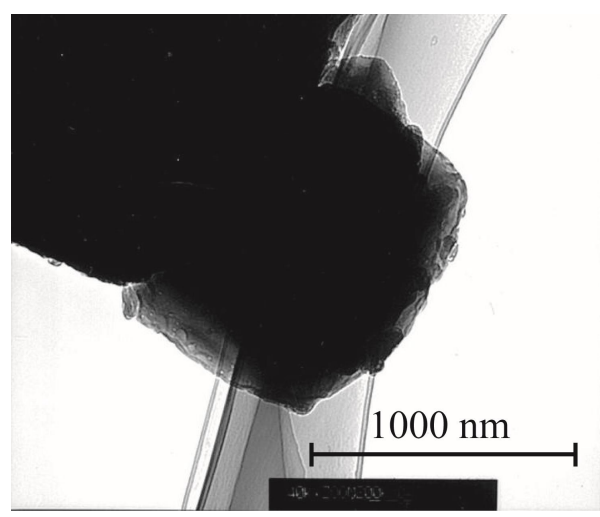

FIG. 5. Transmission electron microscope image of a zeolite sample exposed to indium nanoparticles using a $360 \mu \mathrm{F}$ capacitor. 

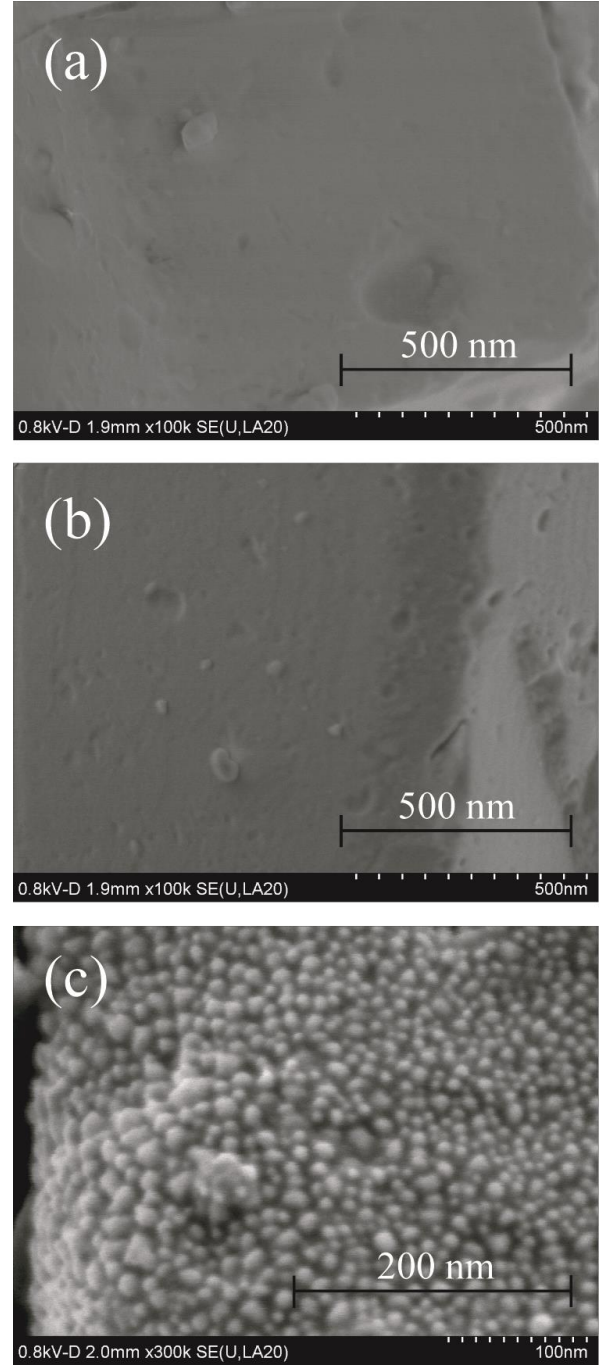

FIG. 6. Scanning electron microscope images of (a) the pristine zeolite sample, and zeolite samples exposed to indium nanoparticles using (b) $360 \mu \mathrm{F}$ and (c) $1080 \mu \mathrm{F}$ capacitors.

high acceleration voltages.

We subsequently attempted to examine samples using SEM but found that even the standard accelerating voltage again damaged the sample. Therefore, the catalysts were observed by SEM using a relatively low accelerating voltage of $800 \mathrm{~V}$ to avoid changing the surface structures. SEM images of the zeolites exposed to indium nanoparticles are presented in Fig. 6(b, c), while Fig. 6(a) shows the pristine material. Both Fig. 6(a) and Fig. 6(b) exhibit similar morphologies, and it is difficult to identify the indium nanoparticles in Fig. 6(b), suggesting that extremely small particles were deposited. In a recent study using gold as the cathode material with similar experimental parameters (such as an electrical capacitance of $360 \mu \mathrm{F}$ ), the mean diameter of gold nanoparticles deposited on the target was found to be $1.3 \mathrm{~nm}[18]$. Therefore, it is reasonable to assume that the indium nanoparticles on sample (b) in the present work could have been less than $10 \mathrm{~nm}$ in diameter.

Although no obvious indium nanoparticles could be seen on the surface of sample (c), the aggregation of nanoparticles is suggested by Fig. 6(c), with aggregate sizes of approximately $10 \mathrm{~nm}$. Thus, both the XPS data

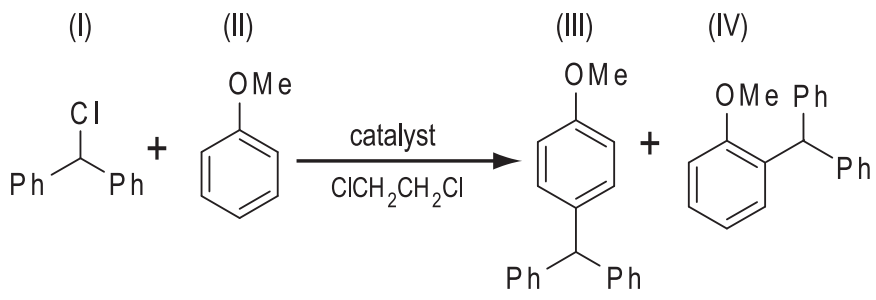

FIG. 7. The Friedel-Crafts alkylation reaction between benzhydryl chloride (I) and anisole (II).<smiles>[CH]</smiles>

(III)

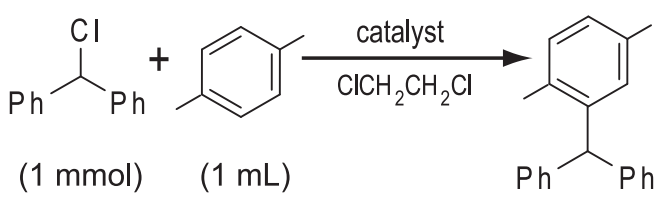

FIG. 8. The Friedel-Crafts alkylation reaction between benzhydryl chloride (I) and xylene (II).

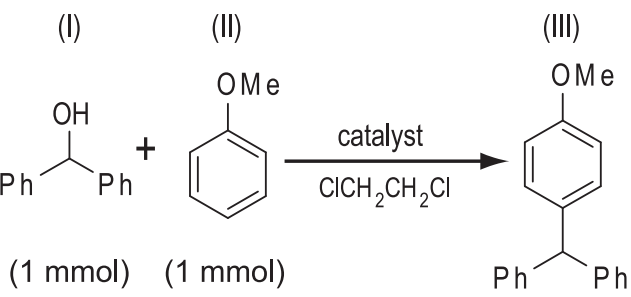

FIG. 9. The Friedel-Crafts alkylation reaction between benzhydryl alcohol (I) and anisole (II).

in Fig. 4 and the SEM image in Fig. 6(c) clearly indicate the presence of indium on the sample surface, suggesting that the deposition of indium on the zeolite succeeded.

The Friedel-Crafts alkylation reaction between benzhydryl chloride and anisole (Fig. 7) [20] was selected as a model reaction to assess the catalytic ability of the new catalyst. In this process, benzhydryl chloride (I) and anisole (II) react to generate products (III) and (IV). In these trials, a portion of the catalyst $(0.05 \mathrm{~g})$ was transferred into a $10 \mathrm{~mL}$, flame-dried, two-necked round bottom flask equipped with a stop cock, a Teflon-coated magnetic stirring bar, and a reflux condenser fitted with a nitrogen inlet adapter. Benzhydryl chloride $(1 \mathrm{mmol})$, anisole $(2 \mathrm{mmol})$ and 1,2-dichloroethane $(2 \mathrm{~mL})$ were added and the resulting mixture was heated to $80^{\circ} \mathrm{C}$ and stirred for $2 \mathrm{~h}$. After completion of the reaction, the mixture was cooled to room temperature and both water $(10 \mathrm{~mL})$ and diethyl ether $(10 \mathrm{~mL})$ were added. The organic layer was separated and the aqueous layer was extracted with two $10 \mathrm{~mL}$ portions of diethyl ether. The combined organic layers were dried over anhydrous magnesium sulfate, filtered, and then concentrated under reduced pressure to yield the crude product. Yields were determined by ${ }^{1} \mathrm{H}$ NMR using an internal standard.

The percentage yields on a molar basis are provided in Table I. These data confirm that the pristine zeolite had no catalytic ability (entry a) because neither product (III) nor (IV) was obtained and $93 \%$ of the original benzhydryl chloride was recovered. In contrast, the indium-impregnated zeolite samples accelerated the 
TABLE I. Results of the Friedel-Crafts alkylation reaction between benzhydryl chloride and anisole (Fig. 7). Yields of products [(III) and (IV)] and recovery of (I) in the presence of (a) the pristine zeolite, and zeolite specimens exposed to indium nanoparticles using (b) $360 \mu \mathrm{F}$ and (c) $1080 \mu \mathrm{F}$ capacitors.

\begin{tabular}{lccc}
\hline \hline \multicolumn{1}{c}{ Sample } & Yield of (III) & Yield of (IV) & Recovery of (I) \\
\hline (a) Pristine zeolite & $0 \%$ & $0 \%$ & $93 \%$ \\
(b) Indium deposited zeolite $(360 \mu \mathrm{F})$ & $75 \%$ & $7 \%$ & $0 \%$ \\
(c) Indium deposited zeolite $(1080 \mu \mathrm{F})$ & $76 \%$ & $7 \%$ & $0 \%$ \\
\hline \hline
\end{tabular}

TABLE II. Results of the Friedel-Crafts alkylation reaction between benzhydryl chloride and xylene (Fig. 8). Yields of product (III) and recovery of (I) in the presence of a zeolite specimen exposed to indium nanoparticles using a $360 \mu \mathrm{F}$ capacitor.

\begin{tabular}{ccc}
\hline \hline Sample & Yield of (III) & Recovery of (I) \\
\hline In-deposited zeolite $(360 \mu \mathrm{F})$ & $0 \%$ & $96 \%$ \\
\hline \hline
\end{tabular}

TABLE III. Results of the Friedel-Crafts alkylation reaction between benzhydryl alcohol and anisole (Fig. 9). Yields of product (III) and recovery of (I) in the presence of a zeolite specimen exposed to indium nanoparticles using a $360 \mu \mathrm{F}$ capacitor

\begin{tabular}{ccc}
\hline \hline Sample & Yield of (III) & Recovery of (I) \\
\hline In-deposited zeolite $(360 \mu \mathrm{F})$ & $0 \%$ & $99 \%$ \\
\hline \hline
\end{tabular}

Friedel-Crafts alkylation. Both catalysts gave similar results, and no benzhydryl chloride was recovered after the reaction (entries b and $c$ ).

The present XPS data suggest that the atomic concentration of indium on sample (c) was higher than that on sample (b) and we therefore anticipated that (c) would show greater catalytic efficiency. However, the data in Table I indicate that the catalytic efficiency of sample (c) was quite similar to that of (b). The reason for this discrepancy is still unknown, although there are two possible explanations. First, the catalytic efficiency might have reached a peak value in sample (b) that could not be increased even with much more indium present. Second, Fig. 6(b, c) shows that the indium nanoparticles on sample (b) were smaller, so the total surface area of the nanoparticles on this material could have been quite high, even though it had less indium.
We subsequently applied sample (b) to the FriedelCrafts alkylation reaction between benzhydryl chloride and xylene (Table II) and between benzhydryl alcohol and anisole (Table III). The equations of these chemical reactions are shown in Figs. 8 and 9, respectively. In both cases, sample (b) showed no catalytic ability and gave none of the product [(III) in Figs. 8 and 9]. These results suggest that the high reactivity of anisole and benzhydryl chloride is necessary for the reaction to proceed, even with the catalyst.

\section{CONCLUSION}

Indium nanoparticles were deposited on zeolite powders using a pulse arc plasma system. The surface characteristics of the resulting samples were assessed via visible absorbance spectroscopy, XRD, XPS, TEM, and SEM. The XPS and SEM results confirmed the successful deposition of indium on the zeolite surfaces, and these materials were found to be capable of catalyzing the Friedel-Crafts alkylation reaction between benzhydryl chloride and anisole. Although the surface concentration of indium and the size of the indium nanoparticles both changed with the capacitance of the plasma system, the catalytic ability of the catalysts synthesized with $360 \mu \mathrm{F}$ and $1080 \mu \mathrm{F}$ capacitors were similar.

\section{ACKNOWLEDGMENTS}

This study was supported by JSPS KAKENHI Grant Number JP15K13406. The authors also wish to thank Prof. S. Hamaguchi (Osaka University), Dr. Y. Agawa (ADVANCE RIKO, Inc.), and Mr. H. Tanaka (ADVANCE RIKO, Inc.) for valuable suggestions and assistance during the plasma experiments.
[1] Y. Onishi, T. Ito, M. Yasuda, and A. Baba, Eur. J. Org. Chem. 1578 (2002).

[2] T. Saito, M. Yasuda, and A. Baba, Synlett 1737 (2005).

[3] U. Schneider and S. Kobayashi, Angew. Chem. Int. Ed. 46, 5909 (2007).

[4] M. Rahman, A. Kr. Bagdi, A. Majee, and A. Hajra, Tetrahedron Lett. 52, 4437 (2011).

[5] P. Gogoi, S. Hazarika, and P. Barman, Sci. Rep. 5, 13873 (2015).
[6] O. Martin, A. J. Martín, C. Mondelli, S. Mitchell, T. F. Segawa, R. Hauert, C. Drouilly, D. Curulla-Ferré, and J. Pérez-Ramírez, Angew. Chem. Int. Ed. 55, 6261 (2016).

[7] S. Yoshimura, K. Hine, M. Kiuchi, Y. Nishimoto, M. Yasuda, A. Baba, and S. Hamaguchi, Appl. Surf. Sci. 257, $192(2010)$

[8] S. Yoshimura, M. Kiuchi, Y. Nishimoto, M. Yasuda, A. Baba, and S. Hamaguchi, Thin Solid Films 520, 4894 (2012). 
[9] S. Yoshimura, K. Ikuse, M. Kiuchi, Y. Nishimoto, M. Yasuda, A. Baba, and S. Hamaguchi, Nucl. Instrum. Methods Phys. Res. B 315, 222 (2013).

[10] S. Yoshimura, M. Kiuchi, Y. Nishimoto, M. Yasuda, A. Baba, Y. Mokuno, S. Sugimoto, and S. Hamaguchi, J. Smart Process. 4, 228 (2015). (in Japanese)

[11] Y. Agawa, H. Tanaka, S. Torisu, S. Endo, A. Tsujimoto, N. Gonohe, V. Malgras, A. Aldalbahi, S. M. Alshehri, Y. Kamachi, C. Li, and Y. Yamauchi, Sci. Technol. Adv. Mater. 16, 024804 (2015).

[12] S-Y. Chun, A. Chayahara, A. Kinomura, N. Tsubouchi, C. Heck, Y. Horino, and H. Fukui, Jpn. J. Appl. Phys. 38, L467 (1999).
[13] S. Yoshimura, Y. Nishimoto, M. Kiuchi, and M. Yasuda, Chem. Lett. 44, 1292 (2015).

[14] S. Yoshimura, Y. Nishimoto, M. Kiuchi, and M. Yasuda, Chem. Lett. 45, 1333 (2016).

[15] S. Yoshimura, Y. Nishimoto, M. Kiuchi, Y. Agawa, H. Tanaka, and M. Yasuda, AIP Adv. 7, 065117 (2017).

[16] Y. Agawa, M. Kunimatsu, T. Ito, Y. Kuwahara, and H. Yamashita, ECS Electrochem. Lett. 4, F57 (2015).

[17] A. Anders and E. Oks, J. Appl. Phys. 101, 043304 (2007).

[18] T. Fujitani, I. Nakamura, T. Akita, M. Okumura, and M. Haruta, Angew. Chem. Int. Ed. 48, 9515 (2009).

[19] A. Ghosh, L. Ma, and C. Gao, J. Mater. Sci. 48, 3926 (2013).

[20] T. Miyai, Y. Onishi, and A. Baba, Tetrahedron Lett. 39, 6291 (1998). 